\title{
Femtosecond laser inscribed Bragg sensor in Terfenol-D coated optical fibre with ablated microslot for the detection of static magnetic fields
}

\author{
G.N. Smith, ${ }^{1 \mathrm{a}}$ T. Allsop, ${ }^{1}$ K. Kalli, ${ }^{2 \mathrm{~b}}$ C. Koutsides, ${ }^{2}$ R. Neal, ${ }^{3}$ K. Sugden, ${ }^{1}$ \\ P. Culverhouse, ${ }^{3}$ and I. Bennion ${ }^{1}$ \\ ${ }^{1}$ Photonics Research Group, Aston University, Birmingham, United Kingdom \\ ${ }^{2}$ Nanophotonics Research Laboratory, Cyprus University of Technology, Lemessos, Cyprus \\ ${ }^{3}$ School of Computing, Communications and Electronics, University of Plymouth, United Kingdom
}

\begin{abstract}
A novel device for the detection and characterisation of static magnetic fields is presented. It consists of a femtosecond laser inscribed fibre Bragg grating (FBG) that is incorporated into an optical fibre with a femtosecond laser micromachined slot. The symmetry of the fibre is broken by the micro-slot, producing non-uniform strain across the fibre cross section. The sensing region is coated with Terfenol-D making the device sensitive to static magnetic fields, whereas the symmetry breaking results in a vectorial sensor for the detection of magnetic fields as low as $0.046 \mathrm{mT}$ with a resolution of $\pm 0.3 \mathrm{mT}$ in transmission and $\pm 0.7 \mathrm{mT}$ in reflection. The sensor output is directly wavelength encoded from the FBG filtering, leading to simple demodulation through the monitoring of wavelength shifts that result as the fibre structure changes shape in response to the external magnetic field. The use of a femtosecond laser to both inscribe the FBG and micro-machine the slot in a single stage, prior to coating the device, significantly simplifies the sensor fabrication.
\end{abstract}

Keywords: Fiber Bragg gratings, optical fiber sensors, femtosecond laser

\section{INTRODUCTION}

Magnetic sensors have been widely used to control and analyse a wide range of devices such as linear and rotary position sensors and current sensors. Optical detection methods that use interferometery [1], Faraday rotation and cantilever arrangements, covering fields from $1 \mathrm{nT}$ to $\sim 1 \mathrm{~T}$, have been used [2-4]. Most reported studies were conducted using high frequency magnetic fields or by using mumetal shields for detecting either slowly varying or static magnetic fields, leading to practical application issues. Therefore, magnetic sensors would greatly benefit from a sensitive optical detection method for which static magnetic fields could be measured and with sensor information that is encoded as an absolute quantity, such as the wavelength encoding offered by a fibre Bragg grating (FBG) sensor. The use of the FBG offers the additional advantages of multiplexing potential, immunity to EM interference and compact dimensions, along with the capability to remotely work in hostile conditions [5]. Here we develop a static magnetic field sensor, exploiting the properties of Terfenol-D, which in the presence of magnetic fields changes dimensions through magnetostriction. [6] Below the Curie temperature, as in this work, the dimensional change is linearly proportional to the magnetic field strength. We utilise the alloying of Dy and $\mathrm{Tb}$, specifically $\mathrm{DyFe}{ }_{2}$ and $T b \mathrm{Fe}_{2}$ having the form $\mathrm{Tb}_{\mathrm{x}} \mathrm{Dy}_{1-\mathrm{x}} \mathrm{Fe}_{2}$, as this gives

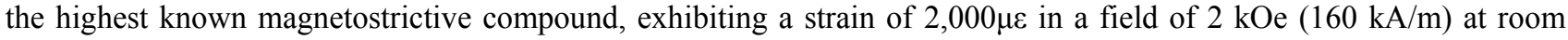
temperature. The novel magnetic sensor uses a femtosecond laser inscribed FBG, combined with a femtosecond machined micro-slot. The micro-slot breaks the fiber symmetry and produces non-uniform strain across the fibre core leading to measurable wavelength shifts as the Terfenol-D coated fibre changes shape in response to the external magnetic field. An important feature of this work is the simplified fabrication of the sensor by using one laser to create the FBG and micro-machined slot. The Terfenol-D when sputtered onto the optical fibre fills the micro-slot creating a robust, monolithic structure. The resulting sensors demonstrate high sensitivity to weak static magnetic fields and sensitivity to the magnetic field direction, which has previously not been demonstrated with comparable approaches.

${ }^{\mathrm{a}}$ smithgn@aston.ac.uk $\quad{ }^{\mathrm{b}}$ kkalli@cytanet.com.cy

21st International Conference on Optical Fiber Sensors, edited by Wojtek J. Bock, Jacques Albert, Xiaoyi Bao, Proc. of SPIE Vol. 7753, 77536N · @ 2011 SPIE · CCC code: 0277-786X/11/\$18 · doi: 10.1117/12.885122 


\section{FABRICATION AND CHARACTERISATION}

The sensor was made using a three step process. A low insertion loss FBG was inscribed point by point [7-8] in Corning SMF-28 using a femtosecond laser (a High Q femtoREGEN laser operating at $1035 \mathrm{~nm}$, pulse duration $300 \mathrm{fs}, 1 \mathrm{kHz}$ pulse repetition rate). A Mitutoyo x50 NIR focusing lens provided a long working distance with a moderate NA thus generating a small and highly accurate focal spot. The accurate nature of the spot placement through the sample due to the large NA and working distance are critical to the direct write nature of the work. The fibre was suspended between fibre mounts on a high precision Aerotech air bearing 2D translation stage, figure 1a. Once the focal spot of the laser beam had been aligned the stage were then translated at a velocity of $1.07 \mathrm{mms}^{-1}$ creating a $2^{\text {nd }}$ order grating with a pitch of $1.07 \mu \mathrm{m}$ over $30 \mathrm{~mm}$ in the centre of the fibre core in order to be resonant at approximately $1550 \mathrm{~nm}$.
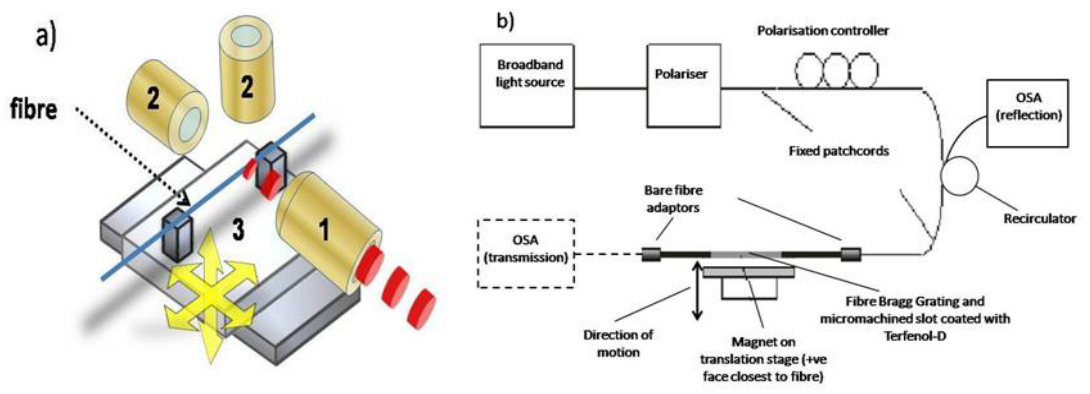

Fig. 1 a) Schematic of the inscription technique, 1 shows the position of the 50x objective lens, 2 the vision optics for alignment and 3 the motion stages, b) Schematic of the characterisation set-up for transmission and reflection for one of the vectors' characterisation.

The second step was to micro-machine the slot, using the same laser and set-up, into the cladding of the fibre directly in line and precisely overlapping the grating. The slots were created using multiple scans along the direction of the fibre axis, with laser pulse energy of $10 \mu \mathrm{J}$. Slot lengths up to $30 \mathrm{~mm}$ long, $15 \mu \mathrm{m}$ wide and $20 \mu \mathrm{m}$ depth were fabricated. Finally, the fiber was RF sputter coated (200W of power), back filling the grooved section, with the alloy Terfenol-D. The fibre was rotated at $60 \mathrm{revs} / \mathrm{min}$, in a vacuum $<10^{-6} \mathrm{mbar}$, with an Argon pressure at $5 \mu \mathrm{m}$ of $\mathrm{Hg}$. The coating thickness was $1 \mu \mathrm{m}$. The relative dimensions of the Terfenol-D monolith to cladding changes the sensitivity of the device, with greater sensitivity achieved with larger monolith. The final device design is shown in the schematic in figure 2.

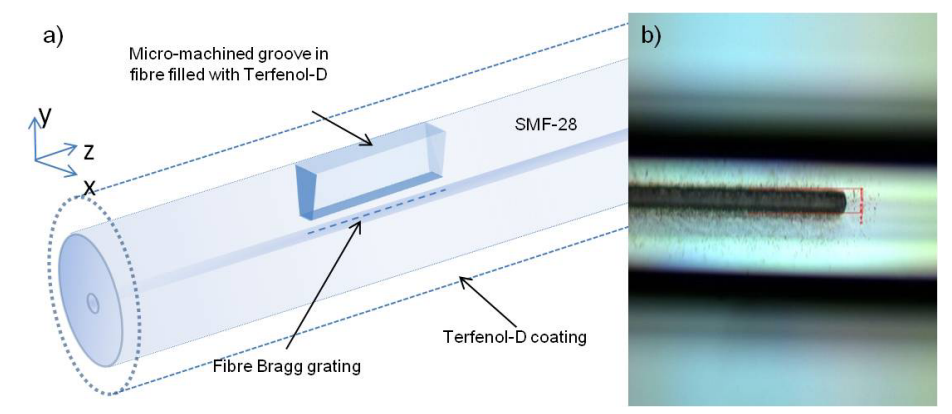

Fig. 2 a) Schematic of the magnetic optical fibre sensor geometry, with femtosecond laser inscribed FBG in the core and micro-slot, all coated with Terfenol-D, b) Microscope image of the slot in the Corning SMF28 prior to being filled and coated.

The sensor was interrogated in transmission and reflection under the variation of the magnetic field strength, figure $1 \mathrm{~b}$. The device was also characterised for its thermal sensitivity using a peltier device. An ELED passed through a polariser (PAT8000B), polarisation controller and coupler prior to illuminating the sample, and the transmission/reflection spectra were monitored using an OSA (Agilent $86142 \mathrm{~B}, 0.06 \mathrm{~nm}$ resolution bandwidth). The static magnetic field was created using a calibrated, permanent magnet mounted on a 3D translation stage that was translated in micron scale increments towards and away from the fibre in the 3 directions indicated in figure $2(x, y, z)$. The spectra were captured at each position and a centroid peak fitting algorithm was used to track the wavelength resonances. The spatial distribution of magnetic flux density generated by the magnets was determined and the results used to determine the field strength at a given separation distance between the sensor and the magnets. 


\section{EXPERIMENTAL RESULTS}

The sensor polarisation dependence was measured in a relative context. Figure 3 shows typical reflection spectra of the complete sensor as a function of varying the polarisation. Polarisation state A showed little sensitivity and is believed to be the state perpendicular to that of the monolith. State $\mathrm{C}$ showed the greatest sensitivity and is believed to be orthogonal to $\mathrm{A}$ and thus in line with the monolith structure. State B is believed to be the resultant of states A and C. The dB drop shows that it is likely to be at slightly less greater $45^{\circ}$ from state $\mathrm{C}$, however, this is expected as the slot and subsequent monolith has physical volume. Figure 4 shows the calculated centroid wavelength and optical strength of the device measured as reflection changes in response to polarisation changes, showing the degree of polarisation dependence of the device, with a $90^{\circ}$ variation between maximum and minimum as would be expected from a device with a slot in one axis.

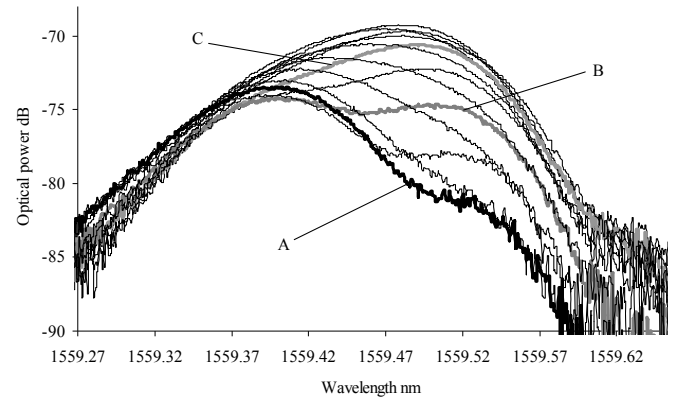

Fig. 3 Polarisation variation in the reflective spectral response of the sensor (coating thickness $1 \mu \mathrm{m}$, length of groove and Bragg

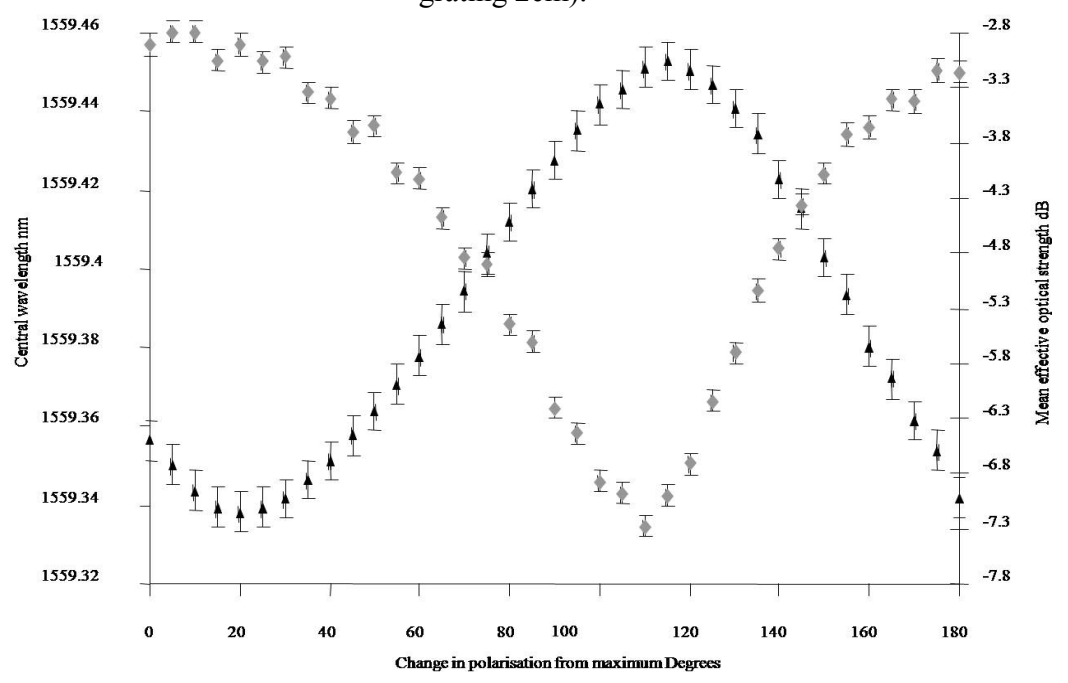

Fig. 4 Variation in the centroid wavelength and effective optical strength of the reflection spectra, as a function of polarisation.

The thermal sensitivity of the device was measured to be $11.4 \mathrm{pm}^{\circ} \mathrm{C}^{-1}$, a typical value for a $\mathrm{FBG}$ in SMF28 and was negated in the magnetic sensitivity experiments by maintaining a constant room temperature. We are currently developing a temperature compensated version of the device. Figure 5 shows transmission measurements using a device with a groove and FBG length of $3 \mathrm{~cm}$. The resulting sensitivities were $0.5 \mathrm{pmmT}^{-1}$ and $0.3 \mathrm{pmmT}^{-1}$ (magnet orientation in figure 1b). There is an increase in sensitivity of the device in transmission that is due to the device length being longer than when examined in reflection after a breakage unrelated to its manufacture. The recorded sensitivity was $\Delta \lambda / \Delta \mathrm{B} \sim$ $0.3 \mathrm{pmmT}^{-1}$ over 22-32 $\mathrm{mT}$. The spectral characteristics were also investigated in a temperature controlled environment, where a standard deviation in wavelength of $\pm 0.014 \mathrm{pm}$ was measured over 5 minutes, and limited by the peltier device. The minimum sensitivity threshold for the temperature stabilised $3 \mathrm{~cm}$ transmission sensor (sensitivity $0.3 \mathrm{pmmT}^{-1}$ ), is $0.047 \mathrm{mT}$. This is greater than the cross-sensitivity between the magnetic and thermal response of the sensor device, at $\sim 3 \times 10^{-3} \mathrm{pm}^{\circ} \mathrm{C}^{-1} \mathrm{~T}^{-1}$. Fitting a linear response to the data of the $3 \mathrm{~cm}$ long fibre sensor a residual average error of $\pm 0.12 \mathrm{pm}$ was seen. This leads to a resolution limit of $\pm 0.3 \mathrm{mT}$ in a magnetic field strength of $\sim 20 \mathrm{mT}$. Any sensor hysteresis may be 
negated by algorithms that compensate for rate independent memory effects. The data presented demonstrates that the response of the sensor is very much dependent upon its separation distance, i.e. field strength, and orientation relative to the magnetic field. This accounts for the differences in sensitivities between the various experiments and shows the expected behaviours of the designed asymmetric sensor geometry.
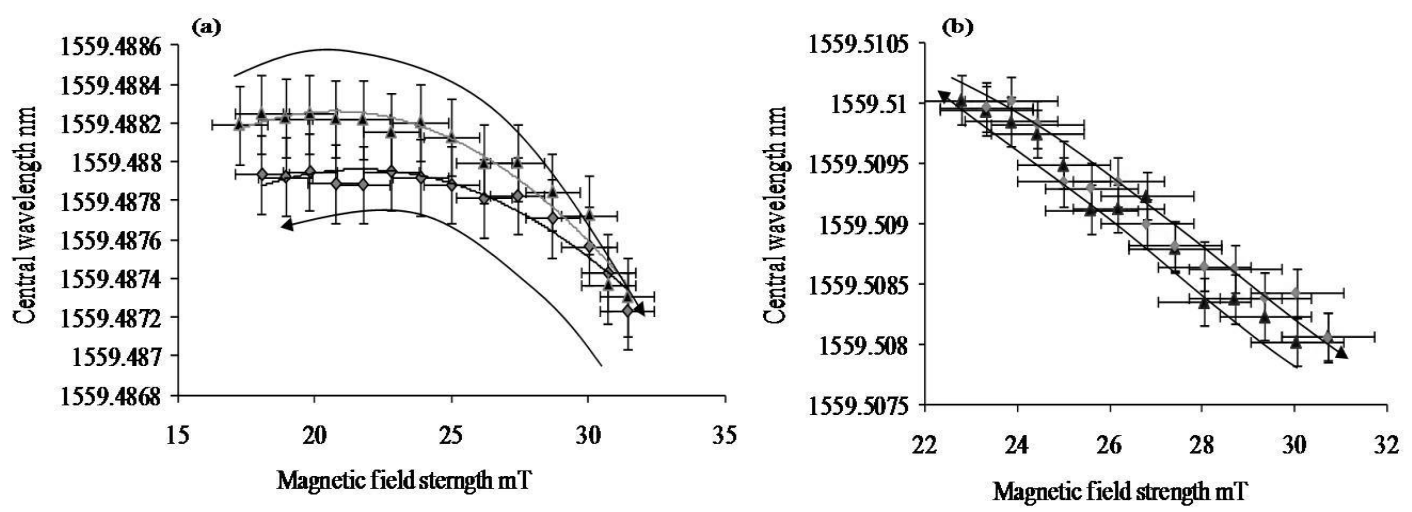

Fig. 5 The spectral characteristics of the device in transmission of the polarisation states of the optical sensor (coating thickness $1 \mu \mathrm{m}$, length of groove and Bragg grating $3 \mathrm{~cm}$ ) as a function of magnetic field strength, (a) for polarisation state A, (b) for polarisation state $\mathrm{C}$ for increasing field strength $(\boldsymbol{\Delta})$ and decreasing field strength $(\diamond)$.

\section{CONCLUSION}

A novel static magnetic field sensor, based on single layer magnetostrictive film of Terfenol-D sputtered onto a single mode fibre with a femtosecond inscribed FBG and slot micromachined into it, is presented. It has demonstrated a minimum detection limit of $0.047 \mathrm{mT}$ and a sensitivity of $0.3 \mathrm{pmmT}^{-1}$ in transmission with a resolution limit of $\pm 0.3 \mathrm{mT}$ in a magnetic field strength of $\sim 20 \mathrm{mT}$. The sensor device clearly demonstrates potential for vectorial field sensing capability. The simplicity of the optical configuration and results show the potential for this technique to be applied to a range of fields due to its small sensor size and ability to be remotely interrogated. This demonstrates the potential for vectorial magnetic field sensing with high sensitivity and large scope for flexibility.

\section{ACKNOWLEDGEMENT}

\section{EU FP7 Project PHOSFOS: “Photonic skins for optical sensing”.}

\section{REFERENCES}

1. W. Xin, C. Shuying, D. Zhigang, W. Xiaoyang, S. Changhai, and C. Jianping, "Experimental Study of Some Key Issues on Fiber-Optic Interferometric Sensors Detecting Weak Magnetic Field," Sensors Journal, IEEE 8, 1173-1179 (2008).

2. $\quad$ L. Sun, S. Jiang, and J. R. Marciante, "All-fiber optical magnetic-field sensor based on Faraday rotation in highly terbium-doped fiber," Optics Express 18, 5407-5412 (2010).

3. P. D. Dinev, "A two-dimensional remote fibre-optic magnetic field and current sensor," Measurement Science \& Technology 7, 1233-1237 (1996).

4. U. Holm, H. Sohlstrom, and T. Brogardh, "Measurement system for magneto-optic sensor materials," Journal of Physics E-Scientific Instruments 17, 885-889 (1984).

5. A. Othonos and K. Kalli, Fiber Bragg Gratings: fundamentals and applications in telecommunications and sensing (Artech House Optoelectronics Library, 1999).

6. M. H. Yang, J. X. Dai, C. M. Zhou, and D. S. Jiang, "Optical fiber magnetic field sensors with TbDyFe magnetostrictive thin films as sensing materials," Optics Express 17, 20777-20782 (2009).

7. M. V. Dubov, M. Amos, K. Igor, and B. Ian, "Point by point FBG inscription by a focused NIR femtosecond laser," in Technical Digest (CD) (Optical Society of America, 2004), CMY6.

8. T. Geernaert, K. Kalli, C. Koutsides, M. Komodromos, T. Nasilowski, W. Urbanczyk, J. Wojcik, F. Berghmans, and H. Thienpont, "Pointby-point fiber Bragg grating inscription in free-standing step-index and photonic crystal fibers using near-IR femtosecond laser," Opt. Lett. 35, 1647-1649 (2010). 